\title{
FANCD2 knockdown with shRNA interference enhances the ionizing radiation sensitivity of nasopharyngeal carcinoma CNE-2 cells
}

\author{
Yilin BAO, Huajun FENG, Feipeng ZHAO, Lijun ZHANG, Shengen XU, Conghong ZHANG, Chong ZHAO, Gang QIN* \\ Department of Otolaryngology-Head and Neck Surgery, The Affiliated Hospital of Southwest Medical University, Luzhou, China \\ ${ }^{*}$ Correspondence: qin-lzm@163.com
}

Received May 11, 2020 / Accepted August 3, 2020

\begin{abstract}
Fanconi anemia complementation group D2 (FANCD2) has been associated with the sensitivity of tumor cells to DNA crosslinking damaging agents in certain solid tumors. However, its role in nasopharyngeal carcinoma (NPC) is still unclear. In the present study, the role of FANCD2 in the response of NPC CNE-2 cells to radiation was investigated. A CNE- 2 cell model with stable FANCD2 silencing was constructed by lentiviral transfection. Fluorescence quantitative PCR and western blotting were used to evaluate FANCD2 expression in CNE-2 cells. The biological impact of FANCD2 silencing on the response of CNE-2 cells to radiation was investigated in vitro and in vivo. The microarray technology, western blotting, and immunohistochemistry were used to analyze the proteins involved in related pathways after irradiation to investigate the underlying mechanism. Lentivirus-mediated shRNA interference stably silenced the FANCD2 gene in CNE-2 cells. In vitro, in the FANCD2-silenced group, cell proliferation was significantly inhibited, apoptosis was increased, and the cell cycle was arrested at the G2/M phase after irradiation. In vivo, FANCD2 silencing slowed tumor growth, as the volume and weight of the xenograft tumors were significantly decreased. Both in vitro and in vivo, the differentially expressed genes NUPR1, FLI1, and FGF21 were downregulated in the FANCD2-silenced group. Our results show that FANCD2 silencing affected the sensitivity of CNE-2 cells to ionizing radiation by regulating cell proliferation, apoptosis, and cell cycle distribution. The mechanism might be associated with changes in NUPR1, FLI1, and FGF21 protein expression due to the FANCD2 silencing. This study provides a promising target for NPC radiotherapy.
\end{abstract}

Key words: Fanconi anemia complementation group D2, Fanconi anemia, nasopharyngeal carcinoma, radiotherapy

Nasopharyngeal carcinoma (NPC) is a malignant tumor that frequently occurs in the nasopharyngeal mucosal epithelium; its incidence is not high worldwide, with an overall incidence lower than $1 / 10,000[1,2]$. In 2012, in total, there were 86,700 new NPC patients and 50,800 deaths worldwide, of which $71 \%$ occurred in Asia [3]. China is among the regions with the highest incidence of NPC, which exhibits regional distribution characteristics; Guangdong, Guangxi, and Fujian are areas with high incidences of NPC. In 2015, there were 60,600 new cases of NPC in China and 34,100 new deaths, and the number of new cases among males was approximately 2.5 times that among females [4]. There is no unified standard for the pathological categorization of NPC [5]. According to the World Health Organization (WHO) standards, NPC can be divided into keratinizing and nonkeratinizing squamous cell carcinoma or differentiated nonkeratinizing carcinoma and nondifferentiated carcinoma. Radiation therapy is the preferred treatment method. However, currently, effective treatment for cases insensitive to radiation therapy or those who relapse after irradiation is lacking. Therefore, exploring a new treatment method that can increase sensitivity to NPC radiotherapy has become a research objective.

Fanconi anemia (FA) is a rare autosomal recessive genetic disease and its main characteristics are progressive bone marrow failure (BMF), endocrine dysfunction, tumor susceptibility, and premature dysfunction of multiple organs. Currently, it is believed that the pathogenesis of FA is associated with DNA damage repair disorders, inflammatory stress, mitochondrial dysfunction, and epigenetic changes [6]. Studies have shown that FA patients are sensitive to ionizing radiation and DNA crosslinkers such as mitomycin $\mathrm{C}$ and cisplatin [7], whose mechanism is to mainly inhibit the repair of DNA strand-crosslinking damage caused by the inactivation of the FA pathway [8]. To date, 22 genes involved in the FA pathway have been discovered, and most encode proteins involved in DNA damage repair, especially the repair of DNA crosslink damage, 
apoptosis, cell cycle regulation, gene transcription, and the maintenance of genome stability. Mutations and deletions in these different genes are also considered the molecular basis of FA development [9-11]. It has been reported that in patients with head and neck squamous cell carcinoma (HNSCC), the mutation rate in the FA pathway is close to $40 \%$ [12]. The risk of HNSCC and gynecological squamous cell carcinoma in adolescents and adults with FA is increased 500 -fold. However, the specific mechanism remains to be elucidated [13]. The monoubiquitination or deubiquitination of FANCD2, which is a key protein in the FA pathway, can lead to FA pathway inactivation, which in turn increases the sensitivity of cells to DNA crosslinking damage [14-16]. Experimental studies involving mice have found that FANCD2 regulates the transcription of stress genes in a DNA-independent manner while enhancing the ability of cells to resist DNA damage [17]. FANCD2-deficient mice exhibit persistent endogenous DNA damage in hematopoietic stem cells and progressive BMF [18]. The upregulation of FANCD2 expression could increase the survival rate of $B R C A 1$ and BRCA2 gene-deficient tumor cells by recruiting alternative DNA damage proteins, changing the cell cycle, and maintaining replication fork stability $[19,20]$. Studies have shown that FANCD2 expression is high in metastatic melanoma [21], colorectal cancer [22], and human glioblastoma [23], and further studies have shown that tumor cells with high FANCD2 expression exhibit proliferation and survival characteristics. However, to date, no studies have investigated FANCD2 and NPC radiotherapy. The aim of this study was to investigate the effect of FANCD2 silencing by short-hairpin RNA (shRNA) interference on the sensitivity of the NPC cell line CNE-2 to radiotherapy and explore the related mechanism to provide new targets and ideas for NPC radiotherapy.

\section{Materials and methods}

Cell lines and cell culture. The NPC cell line CNE-2 was a generous gift from the Department of Otolaryngology-Head and Neck Surgery, Nanfang Hospital, Southern Medical University, China. This study included an experimental group (CNE-2sh: CNE-2 cells with FANCD2 expression silencing using an effective shRNA-FANCD2 interference sequence), a negative control group (CNE-2NC: CNE-2 cells transfected with an ineffective interfering sequence), and a blank control group (CNE-2: wild-type CNE-2 cells without any treatment). The CNE- 2 cells were cultured in Roswell Park Memorial Institute 1640 medium (RPMI-1640; Gibco, USA) supplemented with $10 \%$ fetal bovine serum (FBS; Biological Industries, Israel), $100 \mathrm{U} / \mathrm{ml}$ penicillin, and 100 $\mu \mathrm{g} / \mathrm{ml}$ streptomycin. The cells were maintained in a saturated humidified incubator at a constant-temperature of $37^{\circ} \mathrm{C}$ at $5 \% \mathrm{CO}_{2}$. Puromycin (InvivoGen, San Diego, CA, USA) was added to the experimental group and negative control group as a screening reagent at a final concentration of $2 \mu \mathrm{g} / \mathrm{ml}$.
Construction of the FANCD2 shRNA lentiviral vector and cell transfection. The design of the FANCD2 shRNA interference sequence, the construction of the PLKO.1 vector, and the packaging of the lentivirus particles were completed by Shanghai Zhong Qiao Xin Zhou Biotechnology Co., Ltd., China. The FANCD2 shRNA interference sequence was CCGGCGTCTATTAGATTGGAGGATTCTCGAGAATCCTCCAATCTAATAGACGTTTTTG. The NPC cell line CNE-2 was infected with recombinant lentiviruses and FANCD2 stably silenced cell lines were obtained by continuous selection using puromycin $(2 \mu \mathrm{g} / \mathrm{ml})$.

The relative expression level of FANCD2 was verified by fluorescence quantitative PCR. Total RNA was extracted from three groups of cells according to the instructions of the RNA extraction kit (Tiangen Biotech Co., China). Then, reverse transcription was performed to synthesize cDNA according to the kit instructions (Takara Bio Co., China). FANCD2 gene amplification, to obtain the desired products and data, was performed according to the instructions of the fluorescence quantitative PCR reagent kit (Takara Bio Co., China) as follows: pre-denaturation at $95^{\circ} \mathrm{C}$ for 30 seconds, followed by 40 cycles of $95^{\circ} \mathrm{C}$ for 5 seconds and $60^{\circ} \mathrm{C}$ for 30 seconds. The relative expression levels of FANCD2 mRNA in the three groups of cells were calculated. The primer sequences were as follows: FANCD2 qF: GGAGTCCATGTCTGCTAAAGAG and FANCD2 qR: CAATGTGCTTTAACCGAGTGAG.

Radiation exposure. Cell and animal irradiation were performed using a Siemens linear accelerator (SIEMENS AG, Germany). Irradiation was conducted at room temperature, and the radiation dose rate was $200 \mathrm{cGy} / \mathrm{min}$.

Colony formation assay. A preset concentration of cells in the logarithmic growth phase were seeded in 6-cm dishes, and after $24 \mathrm{~h}$ of incubation, the cells were irradiated $(0,2$, $4,6,8$, and $10 \mathrm{~Gy})$. The cells were continuously cultured for 14 days after irradiation, and the colonies were fixed in $4 \%$ paraformaldehyde, stained with crystal violet, rinsed, and dried. The number of colonies containing $>50$ cells was counted under an inverted microscope. The colony formation rate was calculated (clone formation rate $=$ number of clones/number of cells seeded $\times 100 \%$ ). GraphPad Prism 8 was used for the multi-target single hit model fitting and cell survival curves were plotted.

Cell proliferation analysis. 3-(4,5-dimethylthiazolyl)-2,5-diphenyltetrazolium bromide (MTT; Solarbio Life Science, Beijing, China) was used to evaluate the impact of FANCD2 silencing on the proliferation of CNE-2 cells after irradiation. Cells in the logarithmic growth phase were seeded into 96-well plates at 2000 cells per well. Radiation of 0 or $6 \mathrm{~Gy}$ was administered after cell adhesion. The optical density (OD) values at $450 \mathrm{~nm}$ of the cells in each group were measured $24,48,72$, and $96 \mathrm{~h}$ after irradiation, and cell proliferation curves were plotted.

Cell apoptosis assay. Cells in the logarithmic growth phase in each group were inoculated into 6-well plates at a 
concentration of $1 \times 10^{5}$ cells $/ \mathrm{ml}$. Radiation of 0 or $6 \mathrm{~Gy}$ was administered after cell adhesion. Apoptosis was evaluated at $72 \mathrm{~h}$ after irradiation using the Annexin-V-PE/7AAD apoptosis detection kit according to the manufacturer's instructions (BD Biosciences, USA). Annexin-V staining and 7AAD staining were evaluated as follows: annexin- $\mathrm{V}$ positive $(+)$ and 7AAD negative $(-)$ represented early apoptotic cells (right lower quadrant), while annexin-V $(+)$ and 7AAD $(+)$ represented late apoptotic and necrotic cells (upper right quadrant). The data were analyzed using flow cytometry data analysis.

Cell cycle assay. Cells in the logarithmic growth phase were diluted to a preset concentration as needed and inoculated into $6 \mathrm{~cm}$ dishes. Radiation of 0 or $6 \mathrm{~Gy}$ was administered after cell adhesion. The cells were analyzed by flow cytometry $48 \mathrm{~h}$ after irradiation. The detection methods and procedures were performed according to the manufacturer's instructions of the cell cycle reagent kit (BD Biosciences, USA). The data were statistically processed to obtain the cell cycle phase distribution ratio of the experimental cells.

Animal experiments. BALB/c-nu nude mice (female) aged 3-4 weeks and weighing 10-13 g were purchased from Chengdu DaShuo Biological Technology Co., Ltd., China. The mice were housed in a specific pathogen-free (SPF) experimental animal room at the Department of Oncology, Affiliated Hospital of Southwest Medical University. All animal studies followed the National Institutes of Health Guide for the Care and Use of Laboratory Animals. The nude mice were randomly divided into three groups with four mice per group. The animals were fed for 1 week, after which cells in the exponential growth phase were prepared in PBS to obtain a $1 \times 10^{7} / \mathrm{ml}$ cell suspension and inoculated (using a $1 \mathrm{ml}$ syringe) into the lateral skin on the left posterior thigh of the nude mice. Each nude mouse was subcutaneously injected with a $200 \mu \mathrm{l}$ cell suspension. The xenograft formation rate and tumor formation time per group of nude mice were observed. Once visible xenografts formed, the maximal diameter a) and perpendicular short axis diameter $b$ ) were measured every 3 days, and the tumor volume $\left(\mathrm{V}=\pi / 6 \times a \times b^{2}\right)$ was calculated. On day 22 after the cell inoculation, xenograft irradiation was performed once per day (2 Gy each session). Irradiation continued for 5 days. On the 18th day after the completion of irradiation, all nude mice were sacrificed by cervical dislocation. The nude mice were imaged, the tumor was completely removed, and the xenograft tumor was weighed and imaged. Each xenograft tumor was aliquoted into two portions for subsequent experiments. The animal experiments were performed according to the methods described in previous experiments [24].

DNA microarray technology for the screening of differentially expressed genes. An Agilent SurePrint G3 Human GE $8 \times 60 \mathrm{~K}$ Microarray Kit was used to detect the gene expression differences between the CNE-2sh experimental group and the CNE-2 control group. This experiment was completed by Capital Bio Corporation, Beijing, China. The in vitro amplification and fluorescence labeling were performed using total RNA from cells in each group. After the expression values of all samples were obtained, the data were pre-processed and clustered. A fold ratio difference $\geq 2$ and a p-value $\leq 0.05$ were used as the screening criteria to determine the number of differentially expressed genes. Then, Gene Ontology (GO), Kyoto Encyclopedia of Genes and Genomes (KEGG) pathway, and disease enrichment analyses were performed to analyze the differentially expressed genes. Finally, the differentially expressed genes were introduced into the gene database of the National Center for Biotechnology Information (NCBI) and the PubMed database individually to explore their major functions. After excluding genes that were not related to tumors or had a low-fold differential expression, the candidate genes located downstream of FANCD2 were selected for PCR and western blot validation.

Western blot analysis. A western blot analysis was performed to detect the effect of FANCD2 gene silencing and the protein expression levels of NUPR1, FLI1, and FGF21 in cells and xenograft tumors. The total protein was extracted from the cells and xenograft tumors. The protein samples were adjusted to the same concentration, separated by $12 \%$ sodium dodecyl sulfate polyacrylamide gel electrophoresis (SDS-PAGE), and transferred to a nitrocellulose membrane. The following primary antibodies were used: mouse antihuman monoclonal FANCD2 (sc-20022, 1:300); rabbit antihuman monoclonal FGF21 (sc-81946, 1:1000); rabbit antihuman monoclonal FLI1 (sc-365294, 1:1000); and rabbit anti-human polyclonal p8/NUPR1 (sc-23283, 1:1000) from Santa Cruz Biotechnology, CA, USA. The samples were incubated with the primary antibodies overnight at $4{ }^{\circ} \mathrm{C}$, and the membrane was washed with Tris-buffered saline with Tween-20 (TBST). Then, the following secondary antibodies were added: sheep anti-rabbit secondary antibody (sc-2773, 1:2000) and horseradish peroxidase (HRP)-labelled goat anti-mouse secondary antibody (sc-516102, 1:2000) (Santa Cruz Biotechnology). The samples were incubated at room temperature for $2 \mathrm{~h}$. The membrane was washed with TBST, BeyoECL luminescence solution was added, and the reaction was stopped by adding double-distilled water once the bands appeared in the dark.

Immunohistochemistry. Immunohistochemistry was used to detect the protein expression of NUPR1, FLI1, and FGF21 in the nude mouse xenograft tissues after irradiation. Conventional paraffin-embedded tumor tissues were serially sliced to a thickness of $4 \mu \mathrm{m}$. The sections were conventionally deparaffinized, hydrated, and placed in an ethylenediaminetetraacetic acid (EDTA) solution ( $\mathrm{pH} 9.0)$ and microwaved for antigen retrieval. The sections were immersed in $3 \% \mathrm{H}_{2} \mathrm{O}_{2}$ for $10 \mathrm{~min}$, rinsed three times with PBS, and incubated with primary antibodies at $37^{\circ} \mathrm{C}$ for $1 \mathrm{~h}$. The primary antibodies included an anti-p8 antibody (sc-23283, 1:200), anti-human FGF21 antibody (sc-81946, 1:250), and anti-FLI1 antibody (sc-365294, 1:700) (Santa Cruz Biotechnology). Then, the sections were incubated with a secondary antibody at room 
temperature for $30 \mathrm{~min}$. Diaminobenzidine (DAB) solution was added for color development; the sections were counterstained with hematoxylin, dehydrated and cleared with xylene, and mounted. The expression and localization of each protein in the xenograft tissues were observed under a microscope.

Statistical analysis. The experimental data are presented as the mean $\pm \mathrm{SD}$, and a one-way ANOVA was used for the data comparisons. SPSS 20.0 and GraphPad Prism 8 software were used to analyze the experimental results and plot the curves. A p-value $<0.05$ was considered significant.

\section{Results}

Lentiviral-mediated shRNA interference significantly silenced FANCD2 expression in CNE-2 cells. The gene silencing effect was verified by fluorescence quantitative PCR and western blot analyses. The images were observed under a fluorescence microscope and conventional microscope after transfection (Figure 1A), and the fluorescence quantitative PCR results showed that the expression level of FANCD2 mRNA in the CNE-2sh group was significantly lower than that in the CNE-2 and CNE-2NC groups ( $<<0.01$; Figure $1 \mathrm{~B})$. The western blot analysis results showed that the expression level of FANCD2 protein in the CNE-2sh group was signifi- cantly lower than that in the CNE-2 and CNE-2NC groups and that the silencing efficiency reached $92.20 \% \quad(\mathrm{p}<0.01$; Figures 1C, 1D).

The silencing of FANCD2 expression significantly inhibited the colony formation of CNE-2 cells. The colony formation results showed that the survival fraction in the three groups of cells did not significantly differ before irradiation ( $p>0.05)$, but as the radiation dose increased, the survival fraction in the three groups decreased. The colony formation rate in the CNE-2sh group was significantly lower than that in the control group $(\mathrm{p}<0.05$; Figure 2$)$. The cell survival curves were obtained by multi-target single hit model fitting of the colony formation rate. The results showed that the silencing of FANCD2 expression reduced the survival fraction of the CNE-2sh cells after irradiation.

FANCD2 silencing significantly inhibited the proliferation of CNE-2 cells. The MTT results showed that the proliferation levels in the CNE-2sh group were lower than those in the CNE-2 and CNE-2NC groups at 72 and $96 \mathrm{~h}$ after culture $(\mathrm{p}<0.05$; Figure $3 \mathrm{~A})$. Moreover, the proliferation inhibition effect was enhanced over time after irradiation; the proliferation rate in the CNE-2sh group was significantly lower than that in the control groups after irradiation at the two time-points of 72 and $96 \mathrm{~h}(\mathrm{p}<0.01$; Figure $3 \mathrm{~B})$. Therefore, FANCD2 silencing significantly inhibited the proliferation of
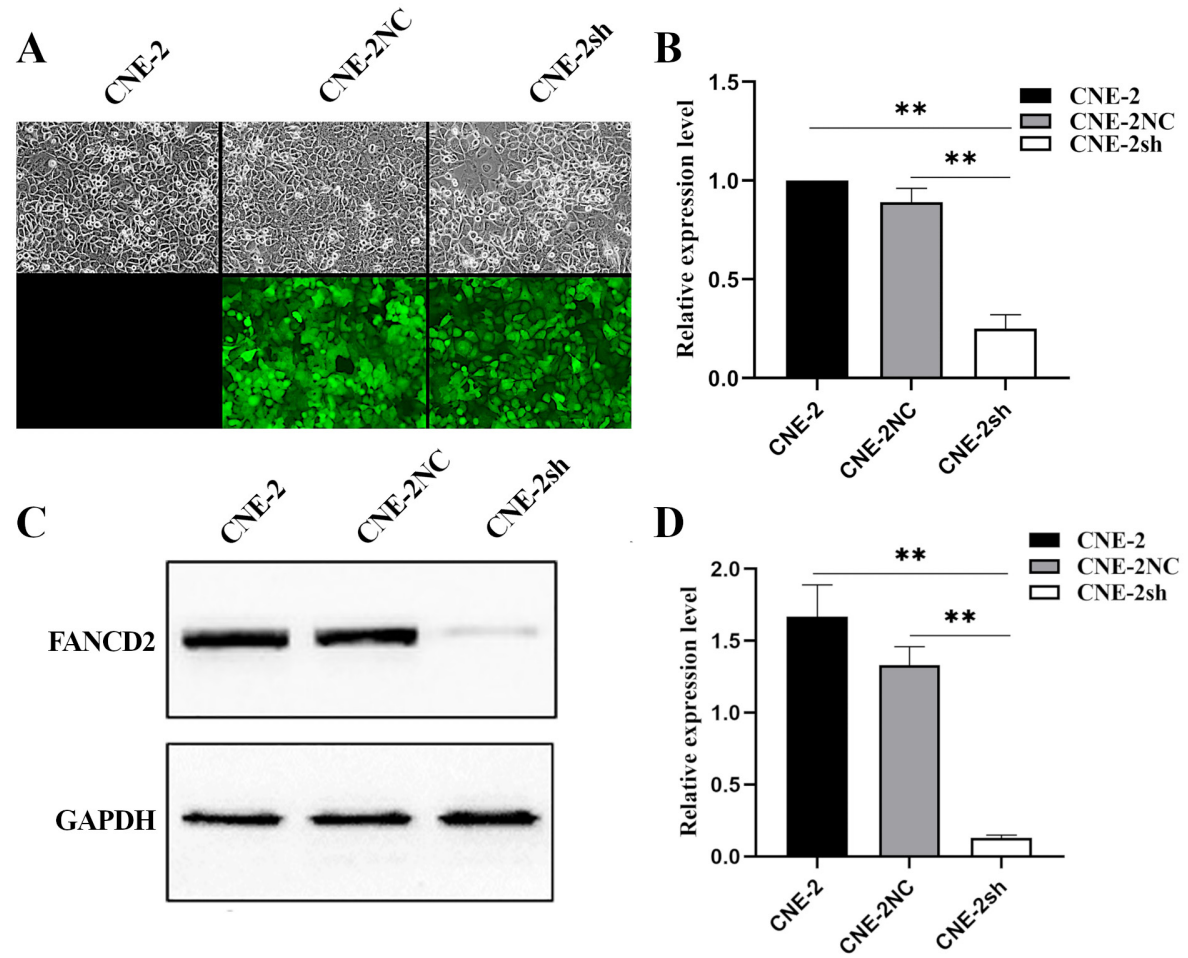

Figure 1. FANCD2 expression in three groups of CNE-2 cells. A) The images were observed under a fluorescence microscope and a conventional microscope after transfection. B) Histogram of FANCD2 mRNA fluorescence quantitative PCR detection results in three groups of cells. C) Western blot analysis showing the FANCD2 protein expression level in the three groups of CNE-2 cells. D) Histogram of FANCD2 relative expression in the three groups of cells. ${ }^{* *} \mathrm{p}<0.01$ 
NPC CNE-2 cells, and the proliferation inhibition effect was time-dependent. In addition, cell proliferation inhibition was enhanced after irradiation.

FANCD2 silencing significantly increased apoptosis in CNE-2 cells and induced cell cycle arrest at the G2/M phase after irradiation. A dose of 6 Gy of radiation was administered to the three groups of cells, and flow cytometry was used to evaluate apoptosis $72 \mathrm{~h}$ after irradiation. The results showed that the CNE-2sh group had a higher apoptosis rate than the CNE-2NC group and CNE-2 group ( $<<0.05$; Figure $4 \mathrm{~A}$ ). Flow cytometry was used to evaluate the cell cycle distribution $48 \mathrm{~h}$ after irradiation. The results showed that the proportion of cells in the G0/G1 phase and S phase in the CNE-2sh group was significantly lower than that in the cells in the CNE-2 and CNE-2NC groups, while the proportion of cells in the G2/M phase was significantly higher than that in the cells in the CNE-2 group and CNE-2NC group $(\mathrm{p}<0.05$; Figure $4 \mathrm{~B})$. These results indicate that FANCD2 silencing significantly increased CNE-2 cell apoptosis after irradiation and induced cell cycle arrest at the G2/M phase.

FANCD2 silencing significantly inhibited the growth of CNE-2 cells in vivo. In each group, xenograft tumors formed and were visible by the naked eye within 5-7 days, and the tumor formation rate was $100 \%$. The average tumor formation time was 6 days, and the tumor formation time did not significantly differ among the three groups of cells (Figure 5A). Before irradiation, on day 22 after inoculation, the tumor volume in the CNE-2sh group was $453.29 \pm 27.31 \mathrm{~mm}^{3}$, which was significantly lower than that in the CNE-2NC group $\left(710.35 \pm 33.23 \mathrm{~mm}^{3}\right)$ and the CNE-2 group $\left(737.78 \pm 23.12 \mathrm{~mm}^{3}\right)(\mathrm{p}<0.01$; Figure $5 \mathrm{~B})$. After irradiation, the volume of the xenograft tumors in the mice in the CNE-2sh experimental group no longer increased, and the final volume was $216.35 \pm 27.75 \mathrm{~mm}^{3}$, which was significantly lower than that of the tumors in the mice in the CNE-2 group $\left(619.68 \pm 37.89 \mathrm{~mm}^{3}\right)$ and $\mathrm{CNE}-2 \mathrm{NC}$ group

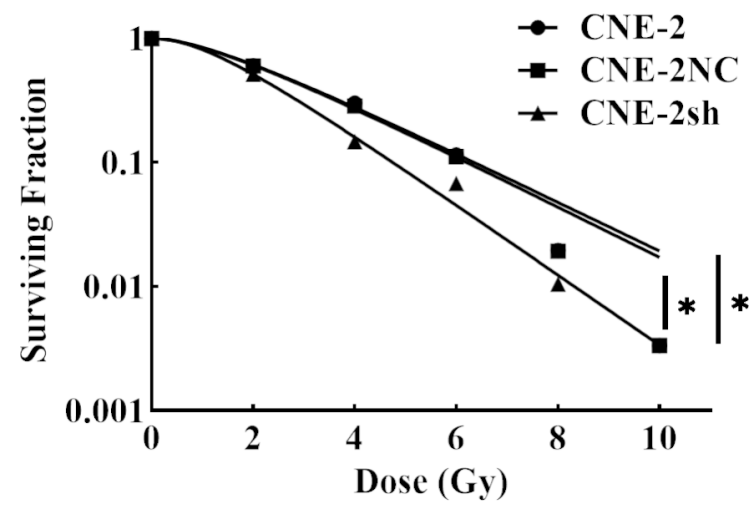

Figure 2. FANCD2 silencing significantly reduced the survival fraction of CNE-2 cells after irradiation. The cell survival fraction in the three groups of cells after 14 days of radiation with $0,2,4,6$, and $8 \mathrm{~Gy} .{ }^{*} \mathrm{p}<0.05$

(577.56 $\pm 27.48 \mathrm{~mm}^{3} ; \mathrm{p}<0.01$; Figures $\left.5 \mathrm{~B}, 5 \mathrm{C}\right)$. In addition, the final weight of the xenograft tumors in the nude mice in the CNE-2sh experimental group after irradiation $(0.33 \pm 0.07 \mathrm{~g})$ was significantly lower than that in the mice in the CNE-2 group $(1.20 \pm 0.28 \mathrm{~g})$ and the CNE-2NC group $(1.10 \pm 0.19 \mathrm{~g}$; $\mathrm{p}<0.01$; Figure $5 \mathrm{D}$ ). These results suggest that FANCD2 silencing inhibits CNE-2 cell growth in vivo and enhances their sensitivity to radiation therapy.

Screening for differentially expressed genes. An Agilent whole human genome microarray was used to detect different gene mRNA expression levels between the CNE-2sh group and CNE-2 group. The results showed that 313 genes were differentially expressed between the two groups of cells, including 193 genes that were upregulated and 120 genes that were downregulated in the CNE-2sh cells (Figure 6, Supplementary Table S1). GO and KEGG (Tables 1, 2) pathway analyses of the differentially expressed mRNAs were
A

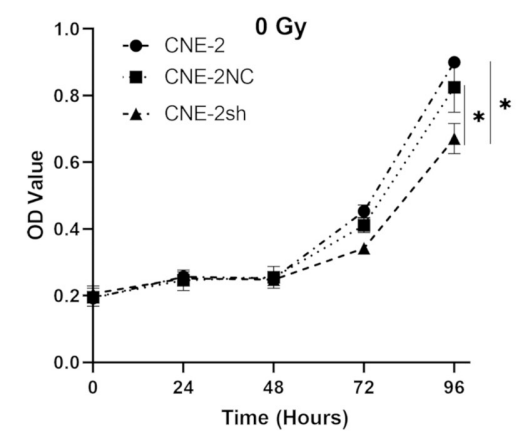

B

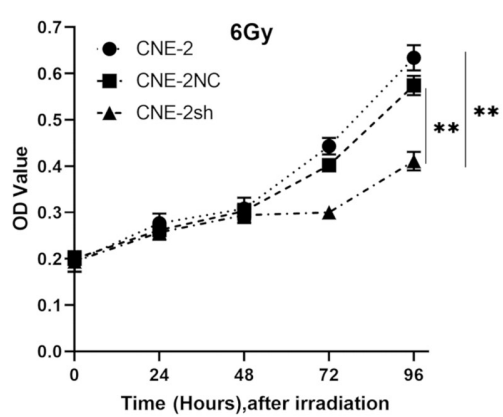

Figure 3. Cell proliferation analysis using an MTT assay. A) At a radiation dose of 0 Gy, the OD values in the experimental group at $72 \mathrm{~h}$ and $96 \mathrm{~h}$ after conventional culture were significantly lower than those in the control group. B) The inhibition effect was more significant in the cells after receiving 6 Gy radiation therapy. FANCD2 silencing significantly inhibited the proliferation of CNE- 2 cells, and the proliferation inhibition effect was time and dose-dependent. ${ }^{*} \mathrm{p}<0.05,{ }^{* *} \mathrm{p}<0.01$ 
A

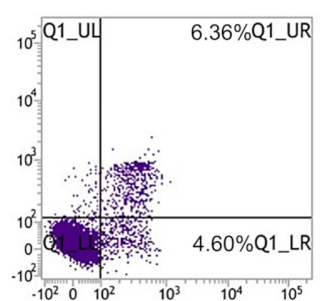

CNE-2

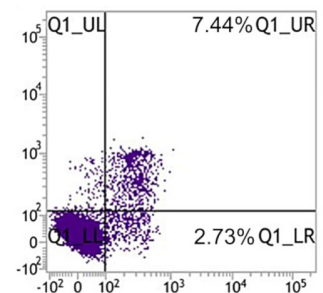

CNE-2NC

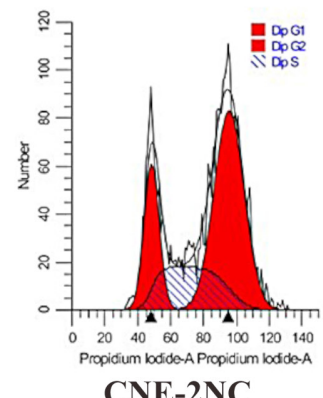

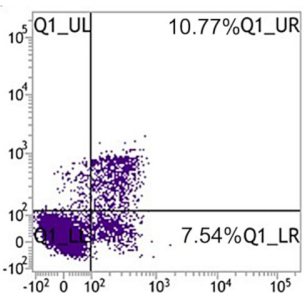

CNE-2sh

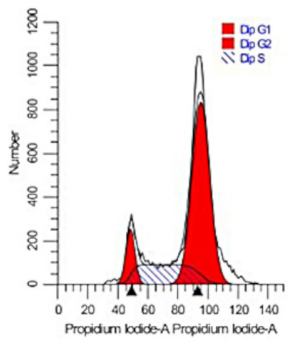

CNE-2sh

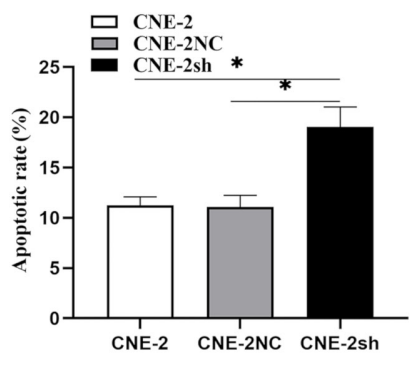

$72 \mathrm{~h}$ after irradiation

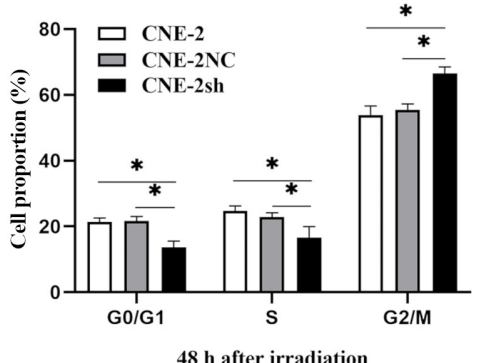

Figure 4. Apoptosis and cell cycle distribution of cells in the three groups after $6 \mathrm{~Gy}$ of radiation. A) Apoptosis plot in which the right upper quadrant represents early apoptotic cells and the right lower quadrant represents late apoptotic and necrotic cells. B) Cell cycle distribution and proportion of cells in different phases. ${ }^{*} \mathbf{p}<0.05$

A

\section{CNE-2 CNE-2NC CNE-2sh}

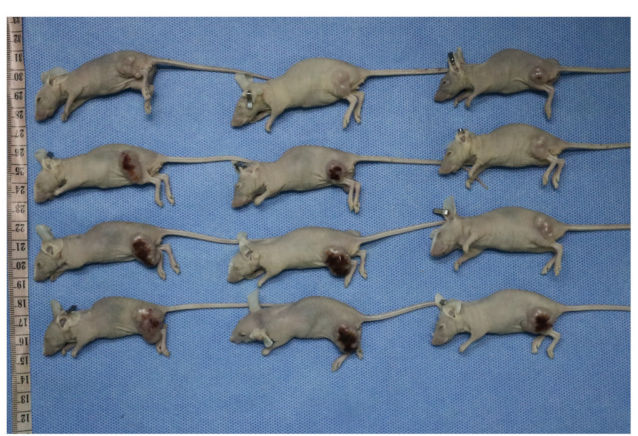

B

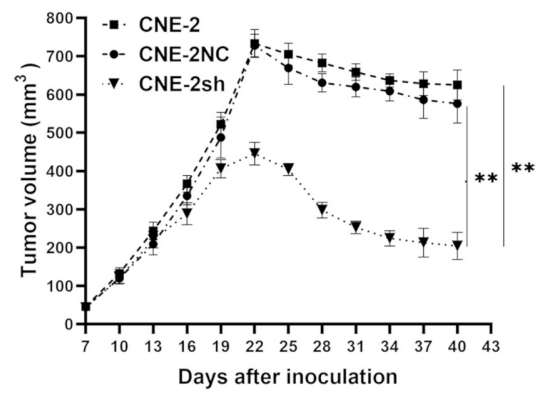

C

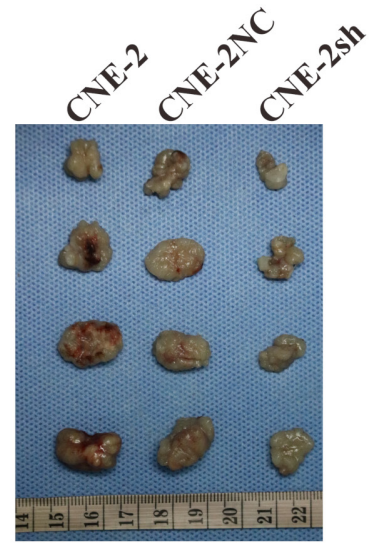

D

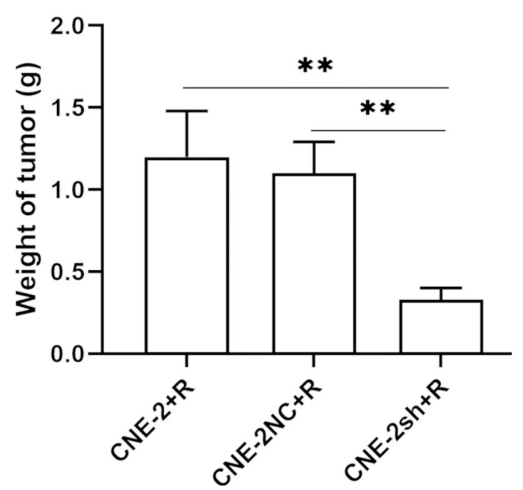

Figure 5. FANCD2 silencing inhibits the growth of xenograft tumors of CNE-2 cells. A) Images of nude mice 40 days after CNE-2 cells were transplanted into the three groups of mice. B) Changes in the tumor volume from 7 days after inoculation to the end of the experiment; irradiation was performed 22 days after inoculation. C) An intact xenograft tumor in a nude mouse. D) Histogram of xenograft tumor weight in nude mice. The xenograft tumor volume and weight in the CNE-2sh group were significantly lower than those in the control group. ${ }^{*} \mathrm{p}<0.05,{ }^{* *} \mathrm{p}<0.01$ 
performed. The differentially expressed genes involved in molecular functions were sorted according to the fold differences, and FGF21, FLI1, and NUPR1 were selected as the top three differentially expressed genes for the subsequent experiments. DNA microarray showed the original signal value of NUPR1, FLI1, and FGF21 in the cells of the experimental group was significantly lower than that in the control groups $(\mathrm{p}<0.05$; Table 3$)$. PCR validation showed that the relative mRNA expression of NUPR1, FLI1, and FGF21 in the cells in the experimental group was significantly lower than that in the control groups $(\mathrm{p}<0.05$; Table 4$)$.

FANCD2 silencing downregulated the FGF21, FLI1, and NUPR1 protein expression levels in CNE- 2 cells. The western blot analysis showed that the FGF21, FLI1, and NUPR1 protein expression levels in the CNE-2sh group were downregulated compared to those in the control CNE-2

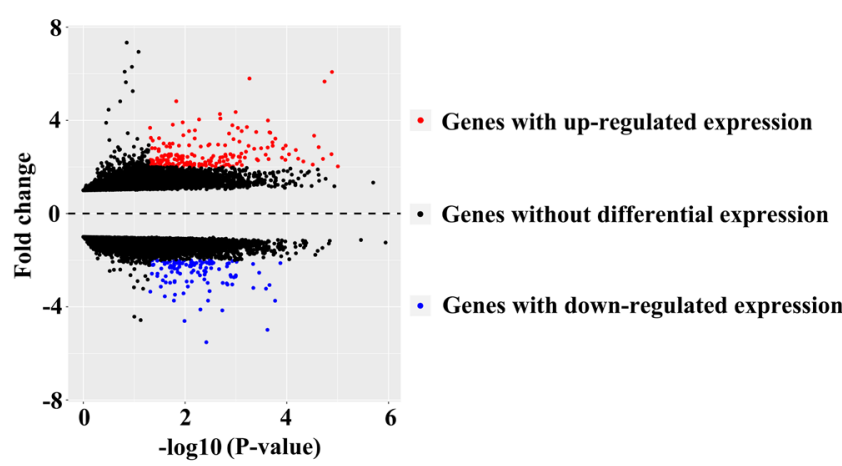

Figure 6. Volcano plot of the differences in gene expression between CNE2 and CNE-2sh cells. Each rectangle in the figure represents the relative expression level of each gene in different samples. The high-expression genes are marked in red, and the low-expression genes are marked in blue.

Table 1. GO enrichment analysis of the differentially expressed genes based on DAVID $6.8(\mathbf{p}<0.05)$.

\begin{tabular}{|c|c|c|c|}
\hline GO Analysis & Enrichment Terms & p-value & Benjamini \\
\hline \multirow[t]{15}{*}{ BP Analysis } & GO:0007165 signal transduction & 0.001 & 0.699 \\
\hline & GO:0010951 negative regulation of endopeptidase activity & 0.002 & 0.619 \\
\hline & GO:0007267 cell-cell signalling & 0.005 & 0.865 \\
\hline & GO:0048546 digestive tract morphogenesis & 0.008 & 0.890 \\
\hline & GO:0032956 regulation of actin cytoskeleton organization & 0.013 & 0.947 \\
\hline & GO:0009615 response to virus & 0.029 & 0.995 \\
\hline & GO:0030574 collagen catabolic process & 0.030 & 0.992 \\
\hline & GO:0070328 triglyceride homeostasis & 0.030 & 0.986 \\
\hline & GO:0060326 cell chemotaxis & 0.031 & 0.979 \\
\hline & GO:0015721 bile acid and bile salt transport & 0.032 & 0.974 \\
\hline & GO:0016477 cell migration & 0.035 & 0.973 \\
\hline & GO:0070374 positive regulation of ERK1 and ERK2 cascade & 0.037 & 0.971 \\
\hline & GO:0070098 chemokine-mediated signalling pathway & 0.039 & 0.965 \\
\hline & GO:2000660 negative regulation of interleukin-1-mediated signalling pathway & 0.041 & 0.965 \\
\hline & GO:0022617 extracellular matrix disassembly & 0.046 & 0.969 \\
\hline \multirow[t]{10}{*}{ CC Analysis } & GO:0005615 extracellular space & 0.000 & 0.009 \\
\hline & GO:0005576 extracellular region & 0.002 & 0.189 \\
\hline & GO:0005578 proteinaceous extracellular matrix & 0.002 & 0.160 \\
\hline & GO:0030175 filopodium & 0.007 & 0.317 \\
\hline & GO:0031410 cytoplasmic vesicle & 0.014 & 0.445 \\
\hline & GO:0005737 cytoplasm & 0.016 & 0.448 \\
\hline & GO:0016328 lateral plasma membrane & 0.019 & 0.447 \\
\hline & GO:0090498 extrinsic component of Golgi membrane & 0.032 & 0.580 \\
\hline & GO:0070062 extracellular exosome & 0.034 & 0.559 \\
\hline & GO:0009925 basal plasma membrane & 0.041 & 0.591 \\
\hline \multirow[t]{5}{*}{ MF Analysis } & GO:0004867 serine-type endopeptidase inhibitor activity & 0.004 & 0.703 \\
\hline & GO:0004896 cytokine receptor activity & 0.006 & 0.655 \\
\hline & GO:0008009 chemokine activity & 0.015 & 0.811 \\
\hline & GO:0005201 extracellular matrix structural constituent & 0.034 & 0.943 \\
\hline & GO:0042169 SH2 domain binding & 0.037 & 0.920 \\
\hline
\end{tabular}


group ( $\mathrm{p}<0.05)$. After irradiation, FGF21, FLI1, and NUPR1 protein expressions were examined again in the cells in the experimental and control groups, and the results showed the same trend; the difference was significant $(\mathrm{p}<0.05$; Figures 7A, 7B). In addition, the western blot and immunohistochemistry analyses of the CNE-2 xenograft tumors after irradiation in the nude mice showed that FGF21, FLI1, and NUPR1 protein expression in the CNE-2sh xenografts after irradiation was significantly downregulated compared to that in the CNE- 2 control group $(\mathrm{p}<0.05$; Figures $7 \mathrm{C}, 7 \mathrm{D}, 8$, and 9). These results are consistent with those of the in vitro cell experiments.

\section{Discussion}

Studies have shown that a functional dysfunction of FANCD2, which is a key protein in the FA pathway, can lead to the inactivation of the FA pathway, which, in turn, increases the sensitivity of cells to DNA cross-linking damage. Li et al. showed that FANCF silencing increased the sensitivity of the anticancer drug mitoxantrone by inhibiting the monoubiquitination of FANCD2 and that the mechanism of its enhanced sensitivity is related to the inhibition of the in vitro proliferation of the breast cancer cell lines MCF-7 and T-47D [25]. The silencing of FANCD2, FANCF, and FANCL by siRNA technology significantly reversed the cisplatin resistance of A549/DDP lung cancer cells and increased their sensitivity to cisplatin treatment by directly inhibiting the FA/BRCA DNA repair pathway, increasing cisplatin-induced DNA

Table 2. KEGG pathway enrichment analysis of the differentially expressed genes based on DAVID $6.8(\mathrm{p}<0.05)$.

\begin{tabular}{lcc}
\hline Enrichment Analysis of KEGG Pathways & p value & Benjamini \\
\hline hsa05146: Amoebiasis & $<0.001$ & 0.017 \\
hsa04060: Cytokine-cytokine receptor interaction & 0.004 & 0.253 \\
hsa04620: Toll-like receptor signaling pathway & 0.005 & 0.228 \\
hsa05202: Transcriptional misregulation in cancer & 0.030 & 0.703 \\
hsa04062: Chemokine signaling pathway & 0.045 & 0.766 \\
\hline
\end{tabular}

damage in lung cancer cells, inhibiting tumor cell proliferation, and promoting apoptosis [26]. Our study results showed that after FANCD2 silencing by shRNA interference, the proliferation activity of CNE-2sh cells in the experimental group was significantly inhibited in vivo and in vitro, and the apoptosis rate increased. These findings indicate that FANCD2 silencing by lentiviral-mediated shRNA interference inhibited the proliferation of NPC CNE- 2 cells and that the radiosensitivity effect of silencing FANCD2 is related to cell proliferation.

Cell cycle regulation plays an important role in tumor cell proliferation, apoptosis, and ionizing radiation sensitization. Pawlik et al. demonstrated that in the DNA damage repair process, cells are arrested in the G2/M phase, and cells in the G2/M phase are more susceptible to the cytotoxic effects of ionizing radiation [27]. The induction of cell cycle arrest at the G2/M phase in tumor cells may be an effective strategy for enhancing the ionizing radiation effect against tumors [28]. Numerous recent studies have shown that increasing radiation-induced G2/M phase arrest in tumor cells can significantly increase the sensitivity of HNSCC, cervical cancer, and prostate cancer cells to ionizing radiation [29-32]. In the present study, the cell cycle detection results after irradiation showed that the proportion of cells in the G2/M phase in the CNE-2sh group was significantly higher than that in the control group cells, indicating that FANCD2 silencing significantly increased radiation-induced G2/M phase cell cycle arrest in the CNE-2 cells and enhanced their ionizing radiation sensitivity. Interestingly, in ionizing radiation sensitivity experiments involving bone marrow stromal cells and IL-3-dependent hematopoietic stem cells of FANCD2-deficient mice, Berhane et al. found that the growth life of bone marrow stromal cells was shortened and showed strong radiation sensitivity and DNA damage involvement. However, there was no obvious radiationinduced G2/M arrest [33]. These results indicate that many factors affect the radiation sensitivity of tumor cells and that different tumor cells may exhibit different responses after irradiation; however, the specific mechanism still requires

Table 3. The relative expression (original signal value) of three differential tumor-associated genes in CNE-2 and CNE-2sh cells detected by a DNA microarray (mean $\pm \mathrm{SD})$.

\begin{tabular}{lcccc}
\hline Gene symbol & CNE-2 (wide type) & CNE-2sh (FANCD2 $2^{-/}$) & T-value & p-value \\
\hline NUPR1 & $13,907.89 \pm 3520.28$ & $3933.00 \pm 509.67$ & 4.857 & $0.037^{*}$ \\
FLI1 & $278.60 \pm 35.72$ & $117.751 \pm 9.07$ & 7.558 & $0.002^{*}$ \\
FGF21 & $663.73 \pm 78.37$ & $143.32 \pm 18.53$ & 11.193 & $0.005^{*}$ \\
\hline
\end{tabular}

${ }^{\star}$ Statistically significant $(\mathrm{p}<0.05)$

Table 4. The relative mRNA expression $\left(2^{-\Delta \Delta C_{9}}\right.$ value) of NUPR1, FLI1 and FGF21 mRNA in each group of cells detected by RT-qPCR (mean \pm SD).

\begin{tabular}{|c|c|c|c|c|}
\hline Gene symbol & CNE-2 (wide type) & 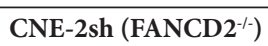 & T-value & p value \\
\hline NUPR1 & $1.000 \pm 0.000$ & $0.148 \pm 0.005$ & 322.026 & $<0.001^{\star}$ \\
\hline FLI1 & $1.000 \pm 0.000$ & $0.434 \pm 0.017$ & 57.568 & $<0.001^{*}$ \\
\hline FGF21 & $1.000 \pm 0.000$ & $0.236 \pm 0.016$ & 84.715 & $<0.001^{*}$ \\
\hline
\end{tabular}

*Statistically significant $(\mathrm{p}<0.05)$ 
A

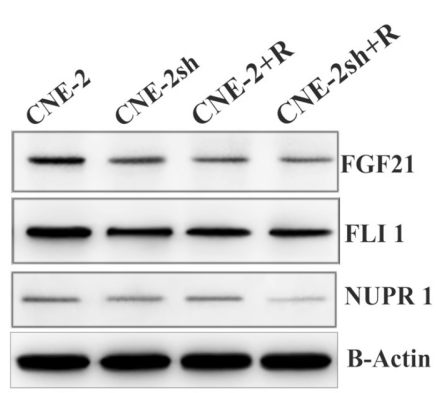

C

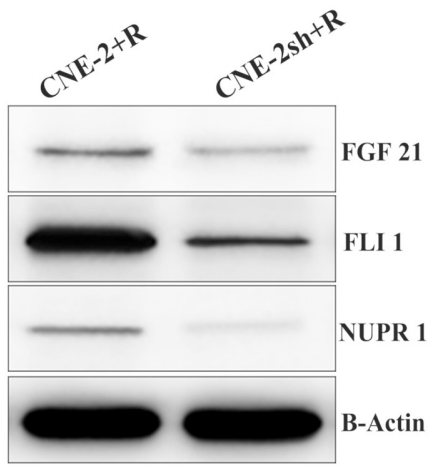

B

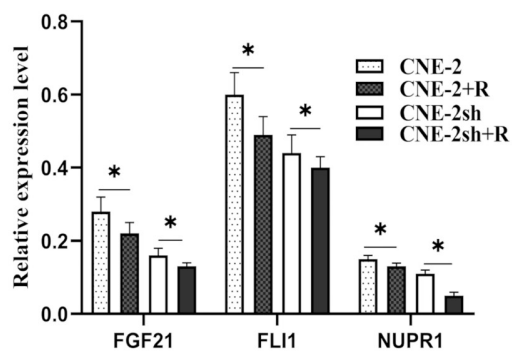

D

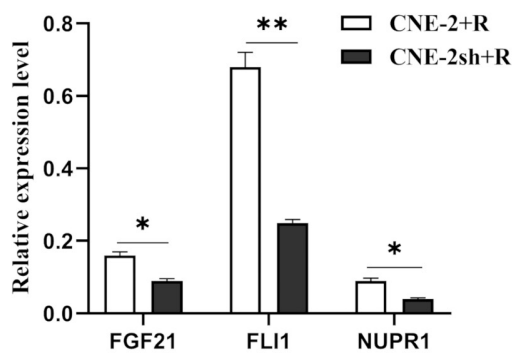

Figure 7. FANCD2 silencing decreases NUPR1, FLI1, and FGF21 protein expression in CNE-2 cells after irradiation in vitro and in vivo. A, B) Detection of FGF21, FLI1, and NUPR1 protein expression in CNE-2sh cells and CNE-2 cells using western blotting before and after irradiation; C, D) Detection of FGF21, FLI1, and NUPR1 protein expression in CNE-2sh xenografts and CNE-2NC xenografts using western blotting. CNE-2+R and CNE-2sh+R represent the radiation exposure group. ${ }^{\star} \mathrm{p}<0.05,{ }^{* *} \mathrm{p}<0.01$

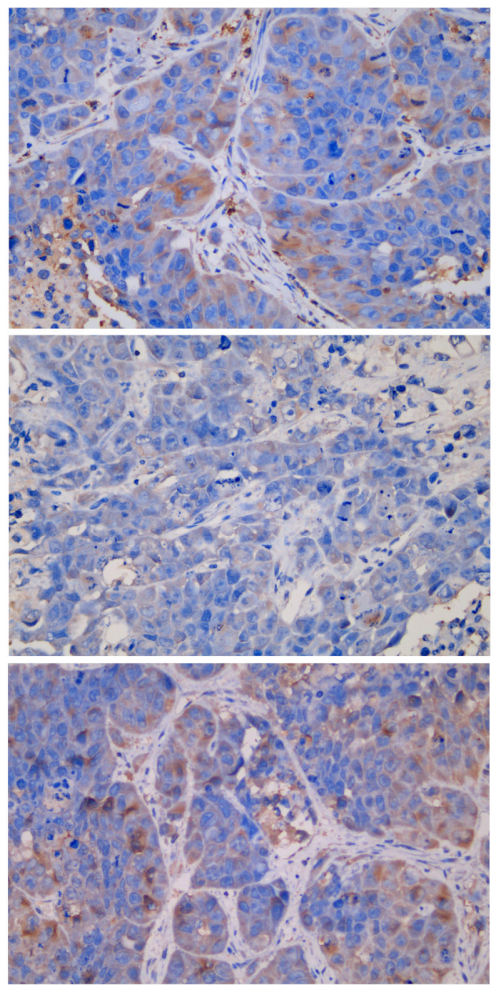

CNE-2+R

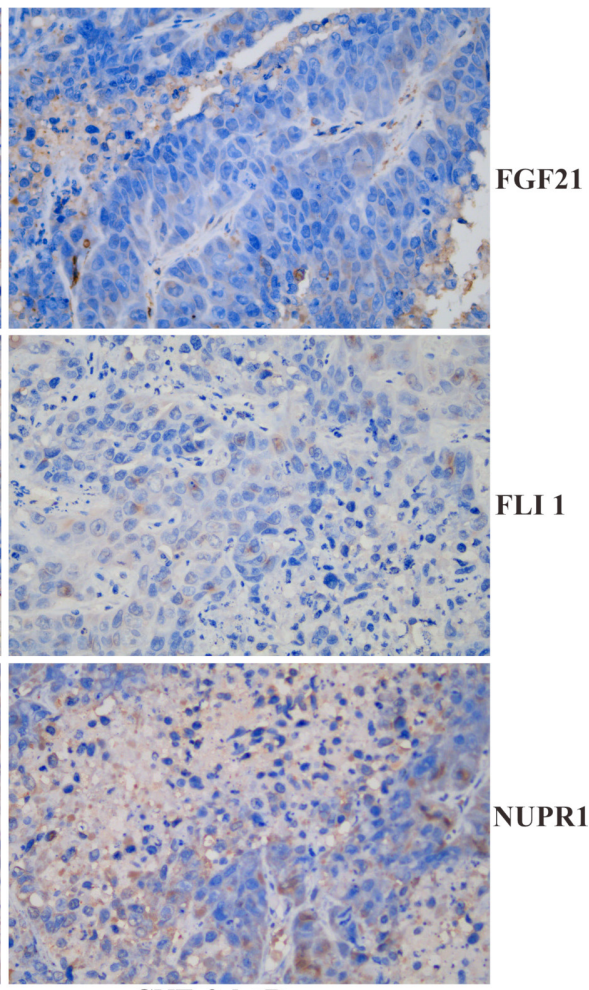

CNE-2sh+R

Figure 8. Immunohistochemistry analysis of FGF21, FLI1, and NUPR1 protein expression in nude mouse xenografts after irradiation. NUPR1 and FLI1 expression were mainly evident in the nucleus and in the cytoplasm. FGF21 expression was mainly located in the cytoplasm (magnification, 400 $\times$ ). 


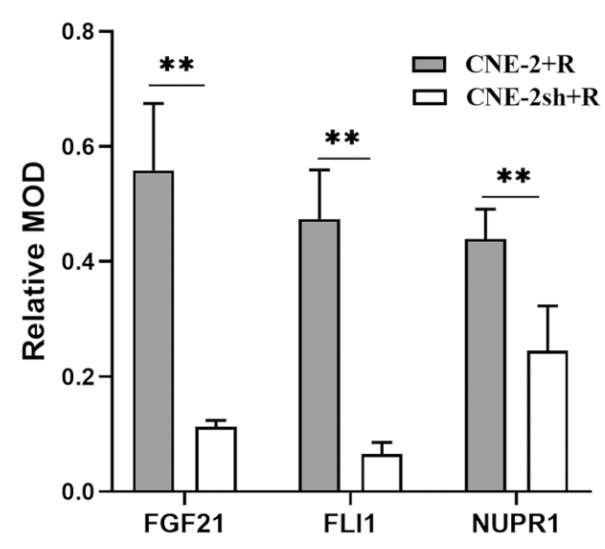

Figure 9. Image-Pro Plus 6 software was used to analyze the relative absorbance values of FGF21, FLI1, and NUPR1 protein in tumors after irradiation. The average absorbance values of FGF21, FLI1, and NUPR1 in the CNE-2sh group were significantly lower than those in the control group CNE-2. ${ }^{*} \mathrm{p}<0.05,{ }^{* *} \mathrm{p}<0.01$

further study. Apoptosis refers to the programmed death of living cells, is regulated by gene expression, and is morphologically different from necrosis. Apoptosis is also an important indicator used to measure the radiation sensitivity of tumor cells. Levine et al. reported that the number of spontaneously apoptotic cells in tumor tissue before irradiation is a powerful predictor of the radiation response [34]. Numerous recent studies have shown that apoptosis is an important factor affecting the ionizing radiation sensitivity of NPC cells $[35,36]$. The flow cytometry analysis in our research demonstrated that FANCD2 silencing increased CNE-2 cell apoptosis after irradiation. These results indicate that silencing FANCD2 gene expression significantly increased radiation-induced apoptosis in CNE-2 cells and enhanced the ionizing radiation sensitivity.

NUPR1 was originally identified as $\mathrm{p} 8$, which is a gene that is preferentially upregulated during the acute inflammatory phase of the cellular stress response in a rat pancreatitis model [37]. Previous studies have confirmed that NUPR1 can significantly promote tumor cell growth. Ree et al. demonstrated that NUPR1 is involved in early breast cancer growth and mediates distant breast cancer metastasis [38]. Recent studies have reported that mitochondrial dysfunction in NUPR1-deficient pancreatic cancer cells leads to increased glycolysis, reduced ATP production, and impaired cellular stress response, thereby promoting programmed cell death [39]. Zeng et al. [40] found that knocking out NUPR1 inhibits the growth of U266 and RPMI8226 multiple myeloma cell lines via the activation of PTEN and caspase-dependent apoptosis. This study found that FANCD2 silencing downregulated NUPR1 expression, inhibited cell proliferation, and promoted apoptosis, however, its specific mechanism needs to be further studied. FLI1 is a member of the ETS transcription factor family and is usually expressed in hematopoietic cells, including most immune-active cells [41], heart cells, lung cells, and ovarian cells $[42,43]$. However, limited attention has been paid to the role of FLI1 in epithelial tumors [44]. The FLI1 gene encodes a transcription factor containing a DNA binding domain, and this gene can be translocated with the Ewing sarcoma (EWS) gene located on chromosome 22 by $\mathrm{T}$ $(11 ; 22)(\mathrm{q} 24 ; \mathrm{q} 12)$ to form a fusion gene EWS-FLI1. Franzetti et al. showed that EWS-FLI1 regulates cell proliferation and migration through a MAPK-independent pathway. High levels of EWS-FLI1 promote ES proliferation, while low levels of EWS-FLI1 drive tumor cell migration [45]. Similarly, in a study investigating the relationship among the glucocorticoid receptor (GR), Ewing's sarcoma and juvenile bone malignant tumors, Srivastava et al. found that GR combined with specific regions of FLI1 can enhance GR transcription activity, thereby promoting tumor proliferation and migration [46]. In addition, studies have shown that the FGF family plays an important role in tumorigenesis and progression. The upregulated expression of various typical FGFs (including FGF1, FGF2, and FGF 6-9) derived from tumor cells or stromal cells can induce the occurrence and progression of various tumors and cancers $[47,48]$. Kang et al. showed that FGF21 was highly expressed in papillary thyroid carcinoma patients' serum and that its expression level was positively correlated with the tumor stage, vascular lymphatic invasion, and recurrence. The underlying mechanism proposed was that FGF21 promotes tumor growth and invasion through the regulation of the signal axis of the FGFR pathway [49]. Studies in China reported that the transient silencing of FGF21 increased the sorafenib-induced inhibition of hepatocellular carcinoma cell proliferation [50]. However, animal experiments have shown that FGF21 is highly expressed in mouse pancreatic acinar cells and that the activation of the proto-oncogene KRAS can significantly reduce FGF21 expression. The injection of recombinant FGF21 can significantly reduce pancreatic inflammation and tumorigenesis, and its specific mechanism is that FGF21 can decrease the ability of RAS to bind guanosine triphosphate. Therefore, it is speculated that FGF21 can be used for the prevention and treatment of pancreatic cancer [51]. The above results indicate that NUPR1, FGF21, and FLI1 promote tumor growth in certain tumors. Interestingly, in this study, the expression levels of NUPR1, FGF21, and FLI1 were all downregulated after FANCD2 silencing. Cell proliferation was inhibited both in vitro and in vivo, apoptosis was increased, and the cell cycle was arrested at the G2/M phase after irradiation, which is consistent with the findings in most studies showing that NUPR1, FGF21, and FLI1 play roles in promoting tumor growth.

In conclusion, the results of this study indicate that FANCD2 silencing can significantly enhance the ionizing radiation sensitivity of NPC CNE-2 cells. We demonstrate that the mechanism underlying the increased radiosensitivity may be related to the regulation of the expression of the proteins NUPR1, FGF21, and FLI1. Our investigation suggests that FANCD2 is a promising target for NPC radiation therapy. 
Supplementary information is available in the online version of the paper.

Acknowledgments: The present study was supported by the Joint Research Project of Science Technology Department of Sichuan Province, Office of Science Technology of Luzhou and Luzhou Medical College (Grant No. 14JC0182), the General Program of National Natural Science Foundation of China (Grant No. 81773529), and the Applied Basic Research Programs Foundation of Sichuan Province (Grant No. 2017JY0109), China.

\section{References}

[1] WEI KR, ZHENG RS, ZHANG SW, LIANG ZH, LI ZM et al. Nasopharyngeal carcinoma incidence and mortality in china, 2013. Chin J Cancer 2017; 36: 90. https://doi. org/10.1186/s40880-017-0257-9

[2] HALESHAPPA RA, THANKY AH, KUNTEGOWDANAHALLI L, KANAKASETTY GB, DASAPPA L et al. Epidemiology and outcomes of nasopharyngeal carcinoma: Experience from a regional cancer center in southern india. South Asian J Cancer 2017; 6: 122-124. https://doi. org/10.4103/2278-330X.214578

[3] TORRE LA, BRAY F, SIEGEL RL, FERLAY J, LORTETTIEULENT J et al. Global cancer statistics, 2012. CA Cancer J Clin 2015; 65: 87-108. https://doi.org/10.3322/caac.21262

[4] CHEN W, ZHENG R, BAADE PD, ZHANG S, ZENG H et al. Cancer statistics in china, 2015. CA Cancer J Clin 2016; 66: 115-132. https://doi.org/10.3322/caac.21338

[5] JI X, ZHANG W, XIE C, WANG B, ZHANG G et al. Nasopharyngeal carcinoma risk by histologic type in central china: Impact of smoking, alcohol and family history. Int J Cancer 2011; 129: 724-732. https://doi.org/10.1002/ijc.25696

[6] BROSH RM, JR., BELLANI M, LIU YSEIDMAN MM. Fanconi anemia: A DNA repair disorder characterized by accelerated decline of the hematopoietic stem cell compartment and other features of aging. Ageing Res Rev 2017; 33: 67-75. https://doi.org/10.1016/j.arr.2016.05.005

[7] TANIGUCHI TD'ANDREA AD. Molecular pathogenesis of fanconi anemia: Recent progress. Blood 2006; 107: 42234233. https://doi.org/10.1182/blood-2005-10-4240

[8] VUNDINTI BR, KORGAONKAR SGHOSH K. Incidence of malignancy and clonal chromosomal abnormalities in fanconi anemia. Indian J Cancer 2010; 47: 397-399. https://doi. org/10.4103/0019-509X.73575

[9] MAMRAK NE, SHIMAMURA AHOWLETT NG. Recent discoveries in the molecular pathogenesis of the inherited bone marrow failure syndrome fanconi anemia. Blood Rev 2017; 31: 93-99. https://doi.org/10.1016/j.blre.2016.10.002

[10] KNIES K, INANO S, RAMIREZ MJ, ISHIAI M, SURRALLES $\mathrm{J}$ et al. Biallelic mutations in the ubiquitin ligase $\mathrm{rfwd} 3$ cause fanconi anemia. J Clin Invest 2017; 127: 3013-3027. https:// doi.org/10.1172/JCI92069

[11] INANO S, SATO K, KATSUKI Y, KOBAYASHI W, TANA$\mathrm{KA} \mathrm{H}$ et al. Rfwd3-mediated ubiquitination promotes timely removal of both rpa and rad51 from DNA damage sites to facilitate homologous recombination. Mol Cell 2017; 66: 622-634 e628. https://doi.org/10.1016/j.molcel.2017.04.022
[12] NALEPA GCLAPP DW. Fanconi anaemia and cancer: An intricate relationship. Nat Rev Cancer 2018; 18: 168-185. https://doi.org/10.1038/nrc.2017.116

[13] KUTLER DI, AUERBACH AD, SATAGOPAN J, GIAMPIETRO PF, BATISH SD et al. High incidence of head and neck squamous cell carcinoma in patients with fanconi anemia. Arch Otolaryngol Head Neck Surg 2003; 129: 106-112. https://doi.org/10.1001/archotol.129.1.106

[14] HAN B, SHEN Y, ZHANG P, JAYABAL P, CHE R et al. Overlooked fancd 2 variant encodes a promising, portent tumor suppressor, and alternative polyadenylation contributes to its expression. Oncotarget 2017; 8: 22490-22500. https://doi. org/10.18632/oncotarget.14989

[15] SHEN Y, ZHANG J, YU HFEI P. Advances in the understanding of fanconi anemia complementation group $\mathrm{d} 2$ protein (fancd2) in human cancer. Cancer Cell Microenviron 2015; 2: e986. https://doi.org/10.14800/ccm.986

[16] CHE R, ZHANG J, NEPAL M, HAN BFEI P. Multifaceted fanconi anemia signaling. Trends Genet 2018; 34: 171-183. https://doi.org/10.1016/j.tig.2017.11.006

[17] PARK E, KIM H, KIM JM, PRIMACK B, VIDAL-CARDENAS S et al. Fancd2 activates transcription of tap63 and suppresses tumorigenesis. Mol Cell 2013; 50: 908-918. https:// doi.org/10.1016/j.molcel.2013.05.017

[18] ATALA A. Re: Endogenous formaldehyde is a hematopoietic stem cell genotoxin and metabolic carcinogen. J Urol 2016; 196: 279-280. https://doi.org/10.1016/j.juro.2016.03.155

[19] KAIS Z, RONDINELLI B, HOLMES A, O'LEARY C, KOZONO D et al. Fancd 2 maintains fork stability in brca1/2-deficient tumors and promotes alternative end-joining DNA repair. Cell Rep 2016; 15: 2488-2499. https://doi.org/10.1016/j. celrep.2016.05.031

[20] MICHL J, ZIMMER J, BUFFA FM, MCDERMOTT UTARSOUNAS M. Fancd2 limits replication stress and genome instability in cells lacking brca2. Nat Struct Mol Biol 2016; 23: 755-757. https://doi.org/10.1038/nsmb.3252

[21] KAUFFMANN A, ROSSELLI F, LAZAR V, WINNEPENNINCKX V, MANSUET-LUPO A et al. High expression of DNA repair pathways is associated with metastasis in melanoma patients. Oncogene 2008; 27: 565-573. https://doi. org/10.1038/sj.onc. 1210700

[22] OZAWA H, IWATSUKI M, MIMORI K, SATO T, JOHANSSON F et al. Fancd 2 mrna overexpression is a bona fide indicator of lymph node metastasis in human colorectal cancer. Ann Surg Oncol 2010; 17: 2341-2348. https://doi. org/10.1245/s10434-010-1002-7

[23] PATIL AA, SAYAL P, DEPONDT ML, BEVERIDGE RD, ROYLANCE A et al. Fancd2 re-expression is associated with glioma grade and chemical inhibition of the fanconi anaemia pathway sensitises gliomas to chemotherapeutic agents. Oncotarget 2014; 5: 6414-6424. https://doi.org/10.18632/oncotarget. 2225

[24] FENG HJ, BAO YL, LIANG ZP, ZHAO FP, XU SE et al. Silencing of fancd 2 enhances the radiosensitivity of metastatic cervical lymph node-derived head and neck squamous cell carcinoma hsc-4 cells. Int J Oncol 2017. https://doi. org/10.3892/ijo.2017.3902 
[25] LI Y, ZHAO L, SUN H, YU J, LI N et al. Gene silencing of fancf potentiates the sensitivity to mitoxantrone through activation of jnk and p38 signal pathways in breast cancer cells. PLoS One 2012; 7: e44254. https://doi.org/10.1371/journal. pone. 0044254

[26] DAI CH, LI J, CHEN P, JIANG HG, WU M et al. Rna interferences targeting the fanconi anemia/brca pathway upstream genes reverse cisplatin resistance in drug-resistant lung cancer cells. J Biomed Sci 2015; 22: 77. https://doi. org/10.1186/s12929-015-0185-4

[27] PAWLIK TMKEYOMARSI K. Role of cell cycle in mediating sensitivity to radiotherapy. Int J Radiat Oncol Biol Phys 2004; 59: 928-942. https://doi.org/10.1016/j.ijrobp.2004.03.005

[28] HEMATULIN A, MEETHANG S, INGKANINAN KSAGAN D. Derris scandens benth extract potentiates radioresistance of hep-2 laryngeal cancer cells. Asian Pac J Cancer Prev 2012; 13: 1289-1295. https://doi.org/10.7314/ apjcp.2012.13.4.1289

[29] LIU J, GE YY, ZHU HC, YANG X, CAI J et al. Fenofibrate increases radiosensitivity in head and neck squamous cell carcinoma via inducing g2/m arrest and apoptosis. Asian Pac J Cancer Prev 2014; 15: 6649-6655. https://doi.org/10.7314/ apjcp.2014.15.16.6649

[30] DENG YR, CHEN XJ, CHEN W, WU LF, JIANG HP et al. $\mathrm{Sp} 1$ contributes to radioresistance of cervical cancer through targeting $\mathrm{g} 2 / \mathrm{m}$ cell cycle checkpoint cdk1. Cancer Manag Res 2019; 11: 5835-5844. https://doi.org/10.2147/CMAR. S200907

[31] CHEN YA, TZENG DTW, HUANG YP, LIN CJ, LO UG et al. Antrocin sensitizes prostate cancer cells to radiotherapy through inhibiting pi3k/akt and mapk signaling pathways. Cancers (Basel) 2018; 11: 34. https://doi.org/10.3390/cancers 11010034

[32] PAN W, GONG S, WANG J, YU L, CHEN Y et al. A nucleartargeted titanium dioxide radiosensitizer for cell cycle regulation and enhanced radiotherapy. Chem Commun (Camb) 2019; 55: 8182-8185. https://doi.org/10.1039/c9cc01651a

[33] BERHANE H, EPPERLY MW, GOFF J, KALASH R, CAO S et al. Radiologic differences between bone marrow stromal and hematopoietic progenitor cell lines from fanconi anemia (fancd2(-/-)) mice. Radiat Res 2014; 181: 76-89. https://doi. org/10.1667/RR13405.1

[34] LEVINE EL, DAVIDSON SE, ROBERTS SA, CHADWICK CA, POTTEN CS et al. Apoptosis as predictor of response to radiotherapy in cervical carcinoma. Lancet 1994; 344: 472. https://doi.org/10.1016/s0140-6736(94)91802-3

[35] HE Y, JING Y, WEI F, TANG Y, YANG L et al. Long noncoding rna pvtl predicts poor prognosis and induces radioresistance by regulating DNA repair and cell apoptosis in nasopharyngeal carcinoma. Cell Death Dis 2018; 9: 235. https://doi.org/10.1038/s41419-018-0265-y

[36] WANG W, LI J, WEN Q, LUO J, CHU S et al. 4egi-1 induces apoptosis and enhances radiotherapy sensitivity in nasopharyngeal carcinoma cells via dr5 induction on 4e-bp1 dephosphorylation. Oncotarget 2016; 7: 21728-21741. https://doi. org/10.18632/oncotarget.7824
[37] MALLO GV, FIEDLER F, CALVO EL, ORTIZ EM, VASSEUR $S$ et al. Cloning and expression of the rat $\mathrm{p} 8 \mathrm{cdna}$, a new gene activated in pancreas during the acute phase of pancreatitis, pancreatic development, and regeneration, and which promotes cellular growth. J Biol Chem 1997; 272: 32360-32369. https://doi.org/10.1074/jbc.272.51.32360

[38] REE AH, PACHECO MM, TVERMYR M, FODSTAD OBRENTANI MM. Expression of a novel factor, com1, in early tumor progression of breast cancer. Clin Cancer Res 2000; 6: 1778-1783.

[39] SANTOFIMIA-CASTANO P, LAN W, BINTZ J, GAYET O, CARRIER A et al. Inactivation of nupr1 promotes cell death by coupling er-stress responses with necrosis. Sci Rep 2018; 8: 16999. https://doi.org/10.1038/s41598-018-35020-3

[40] ZENG C, LI X, LI A, YI B, PENG X et al. Knockdown of nupr1 inhibits the growth of u266 and rpmi8226 multiple myeloma cell lines via activating pten and caspase activation dependent apoptosis. Oncol Rep 2018; 40: 1487-1494. https://doi.org/10.3892/or.2018.6544

[41] SUZUKI E, WILLIAMS S, SATO S, GILKESON G, WATSON DK et al. The transcription factor fli-1 regulates monocyte, macrophage and dendritic cell development in mice. Immunology 2013; 139: 318-327. https://doi.org/10.1111/ imm. 12070

[42] BEN-DAVID Y, GIDDENS EBBERNSTEIN A. Identification and mapping of a common proviral integration site fli- 1 in erythroleukemia cells induced by friend murine leukemia virus. Proc Natl Acad Sci U S A 1990; 87: 1332-1336. https:// doi.org/10.1073/pnas.87.4.1332

[43] BEN-DAVID Y, GIDDENS EB, LETWIN KBERNSTEIN A. Erythroleukemia induction by friend murine leukemia virus: Insertional activation of a new member of the ets gene family, fli-1, closely linked to c-ets-1. Genes Dev 1991; 5: 908-918. https://doi.org/10.1101/gad.5.6.908

[44] SCHEIBER MN, WATSON PM, RUMBOLDT T, STANLEY C, WILSON RC et al. Fli1 expression is correlated with breast cancer cellular growth, migration, and invasion and altered gene expression. Neoplasia 2014; 16: 801-813. https://doi. org/10.1016/j.neo.2014.08.007

[45] FRANZETTI GA, LAUD-DUVAL K, VAN DER ENT W, BRISAC A, IRONDELLE $M$ et al. Cell-to-cell heterogeneity of ewsr1-fli1 activity determines proliferation/migration choices in ewing sarcoma cells. Oncogene 2017; 36: 35053514. https://doi.org/10.1038/onc.2016.498

[46] SRIVASTAVA S, NATARAJ NB, SEKAR A, GHOSH S, BORNSTEIN C et al. Ets proteins bind with glucocorticoid receptors: Relevance for treatment of ewing sarcoma. Cell Rep 2019; 29: 104-117 e104. https://doi.org/10.1016/j.celrep.2019.08.088

[47] TURNER NGROSE R. Fibroblast growth factor signalling: From development to cancer. Nat Rev Cancer 2010; 10: 116129. https://doi.org/10.1038/nrc2780

[48] ACEVEDO VD, GANGULA RD, FREEMAN KW, LI R, ZHANG $Y$ et al. Inducible fgfr- 1 activation leads to irreversible prostate adenocarcinoma and an epithelial-to-mesenchymal transition. Cancer Cell 2007; 12: 559-571. https:// doi.org/10.1016/j.ccr.2007.11.004 
[49] KANG YE, KIM JT, LIM MA, OH C, LIU L et al. Association between circulating fibroblast growth factor 21 and aggressiveness in thyroid cancer. Cancers (Basel) 2019; 11: 1154. https://doi.org/10.3390/cancers11081154

[50] LIU M, ZHOU R, WU X, XU X, SU M et al. Clinicopathologic charcterization of sorafenib-induced endoplasmic reticulum stress in human liver cancer cells. J Physiol Pharmacol 2018; 69: 4. https://doi.org/10.26402/jpp.2018.4.08
[51] LUO Y, YANG Y, LIU M, WANG D, WANG F et al. Oncogenic kras reduces expression of fgf2 1 in acinar cells to promote pancreatic tumorigenesis in mice on a high-fat diet. Gastroenterology 2019; 157: 1413-1428 e1411. https://doi. org/10.1053/j.gastro.2019.07.030 